\title{
Christoph Markschies \\ Wissen in der Zeit \\ Drei kurze Erwägungen zu Fortschritten, Rückschritten und Stagnationen in der Wissenschaft
}

So lange über Wissen und Wissenschaft nachgedacht wird, so lange reden die einen von der immanenten Progressivität des Wissens und klagen die anderen über immanente Stagnation und implizierten Rückschritt: „Es geschieht nichts Neues unter der Sonne", lautet eine frühe und oft zitierte Formulierung dieser pessimistischen Sicht auf die Entwicklung des Wissens ${ }^{1}$. In gegenwärtigen Zeiten der neuen Unübersichtlichkeit gerade auch des Wissens haben natürlich nicht nur die Fortschrittsoptimisten Hochkonjunktur, sondern genauso auch die Verfallspessimisten. „Wir leben bereits in den barocken Vorstädten der Glaubensgesellschaft. Die mythischen Brücken waren nicht wirklich abgebrochen" - mit diesen Worten hat vor einiger Zeit ein Festredner einen Kongreß für Philosophie eröffnet und damit das Modell eines beständig fortschreitenden Weges in das Paradies der Wissensgesell-

1 Kohelet 1,9; vgl. dazu O. Kaiser, Der Mensch unter dem Schicksal, in: ders., Der Mensch unter dem Schicksal. Studien zur Geschichte, Theologie und Gegenwartsbedeutung der Weisheit (BZAW 161), Berlin/New York 1985, S. 63-90. - Meiner Mitarbeiterin Charlotte Köckert danke ich ganz herzlich für die Bereitstellung von Literatur zum Thema; die Vortragsfassung für das Symposium wurde lediglich durch einige Fußnoten und Nachweise ergänzt. 
schaft problematisiert ${ }^{2}$. Die berühmte und nicht minder alte Debatte über die Grenzen des Wissens wirkt angesichts solcher schroffer Alternativen, angesichts von angestrengtem Fortschrittsoptimismus und leicht resigniertem Verfallspessimismus fast wie ein Aufruf zu pragmatischer Nüchternheit: Jürgen Mittelstraß fragte beispielsweise vor kurzem: „Gibt es Grenzen des Wissens?" und beantwortete diese Frage mit einer ebenso klaren wie vermittelnden These ${ }^{3}$ : Gerade die prinzipielle Offenheit des Wissens, die seine theoretische Grenzenlosigkeit ausmache, impliziere praktische Grenzen des Wissen, nämlich Irrtumsgrenzen (,der wissenschaftliche Verstand verrennt sich in seinen eigenen Unzulänglichkeiten"), ökonomische Grenzen und ethische Grenzen.

Von einem Historiker, der sich hauptsächlich mit der christlichen Antike beschäftigt, werden Sie heute nachmittag hoffentlich keinen eigenständigen neuen Beitrag zu dieser langen Debatte über die Grenzen des Wissens erwarten, beispielsweise keine erneute Kommentierung von Reschers Thesen über wissenschaftlichen Fortschritt ${ }^{4}$, keine vertrauten Einwände gegen Thomas Kuhns Modell wissenschaftlicher Revolutionen ${ }^{5}$ oder eine mehr oder weniger ausführliche Nachzeichnung der eben angespielten These von Mittelstraß. Ich möchte vielmehr bei meinem Leisten bleiben und in Fortsetzung des schönen Referates von Volker Gerhardt fragen, ob wir jene gegenwärtige De-

2 D. Simon, Die Glaubensgesellschaft, in: Die Zukunft des Wissens. XVIII. Deutscher Kongreß für Philosophie, Konstanz, 4.-8. Oktober 1999. Vorträge und Kolloquien, hg. v. J. Mittelstraß, Berlin 2000, (S. 24-33) hier S. 33.

3 Gibt es Grenzen des Wissens?, in: ders., Wissen und Grenzen. Philosophische Studien (STW 1566), Frankfurt/M. 2001, (S. 120-137) hier S. 137: „Das aber heißt, die Wissenschaft hat sehr wohl praktische, aber keine theoretischen Grenzen. Und das macht ineins das Menschliche und das Göttliche an der Wissenschaft aus".

4 N. Rescher, Wissenschaftlicher Fortschritt. Eine Studie über die Ökonomie der Forschung (de Gruyter Studienbuch), Berlin/New York 1982.

5 Th.S. Kuhn, Die Struktur wissenschaftlicher Revolutionen (stw 25), Frankfurt/M. ${ }^{14} 1997$ (= The Structure of Scientific Revolutions, Chicago ${ }^{21970)}$. 
batte über Fortschritt und Rückschritt im Prozeß der Gewinnung des Wissens präziser führen könnten, wenn wir uns an vergangene Gestalten dieser Debatte erinnern, uns insbesondere daran erinnern, daß das Wissen und die darauf bezogene Wissenschaft ${ }^{6}$ ja nicht erst auf diesem Symposium im Horizont ihrer Zeitlichkeit thematisiert werden. Ich gestehe dabei gern, überhaupt auf die Idee zu einem solchen Vortrag gekommen zu sein, weil Emil du Bois-Reymond in seiner berühmten Rede über „Die sieben Welträtsel“ am Leibniztag $1880^{7}$ nicht nur vier (seiner Ansicht nach) jeder Erkenntnis schlechterdings transzendente und daher unlösbare Welträtsel vorstellte (auf die jüngst noch einmal Wolf Singer am Leibniztag des Jahres 2000 Bezug nahm ${ }^{8}$ ), sondern im selben Atemzug jeden Originalitätswert für diese Erkenntnis bestritt. Der Festredner wörtlich: „So schmeichelhaft es mir war, meine Darlegung (sc. in der berühmten Vorlesung „Über die Grenzen des Naturerkennens“ von 1872) als Kantsche Tat gepriesen zu sehen, ich muß diesen Ruhm zurückweisen. Wie bemerkt, meine Aufstellungen enthielten nichts, was bei einiger Belesenheit in älteren philosophischen Schriften nicht jedem bekannt sein konnte, der sich darum kümmerte. Aber seit der Umgestaltung der Philosophie durch Kant hat diese Disziplin einen so esoterischen Charakter angenommen $(\ldots)^{\text {“ }}$.

6 Wissenschaft verstehen wir dabei als einen Versuch, nach bestimmten verbindlichen Methoden und Regeln Wissen zu generieren und zu bewahren.

7 E. du Bois-Reymond, Vorträge über Philosophie und Gesellschaft, eingel. u. mit erklärenden Anmerkungen hg. v. S. Wolgast (PhB 287), Hamburg 1974, (S. 159-187) hier S. 160. - Vgl. den Bezug bei W. Singer, Ignorabimus? Ignoramus. Wie das Bewußtsein in die Welt kam, in: Berlin-Brandenburgische Akademie der Wissenschaften. Jabrbuch 2000, Berlin 2001, (S. 115-124) hier S. 124: „Weil wir heute wissen, daß wir nicht wissen, wo uns die Grenzen des Wißbaren gezogen sind, würde ich vorschlagen, Du Bois-Reymond's ignorabimus durch ein schlichtes ignoramus zu ersetzen“.

8 Nämlich: das Wesen von Kraft und Materie, den Ursprung der Bewegung, die Entstehung von Empfindung, Bewußtsein und Willensfreiheit. 
Ich werde im Folgenden daher versuchen, einige maßgebliche antike Stimmen zu Gehör zu bringen, die die Frage nach einer immanenten Progressivität des Wissens im Horizont der Zeitlichkeit verhandelt haben. Dabei beschränke ich mich weitgehend auf pagane Stimmen. Denn erst einmal liegt natürlich der Verdacht nahe, daß die christlichen Denker der Antike solche Fragen im engen Korsett einer strikten heilsgeschichtlichen Dynamik und damit nach stets demselben Grundschema behandelten. Außerdem ist mindestens nicht ausgeschlossen, daß dabei nicht selten die Semantik einer Rede von engen Grenzen geschöpflicher Erkenntnis durch die Pragmatik, mit der für dieses Konzept schlechthinnige, also grenzenlose Autorität beansprucht wurde, dementiert wurde. Exakt diesen Vorbehalt gegen die christliche Theoriebildung kann man übrigens wunderbar bei du Bois-Reymond spüren, wenn er am Leibniztag 1880 sagte, sein berühmtes „Bekenntnis des ,Ignorabimus““ schmecke „fast nach konsistorialrätlicher Demut" "9. Und mit einer solchen Form der Demut - und käme sie im historischen Gewande daher möchte ich Zuhörer wie Leser dann doch eher nicht behelligen.

\section{Eine Erwägung zum Fortschrittsoptimismus}

Ich beginne unseren schon aus Zeitgründen sehr fragmentarischen Erkundungsgang durch antike Debatten über die immanente Progressivität des Wissens im Horizont der Zeitlichkeit bei einem sehr frühen griechischen Denker, bei dem Vorsokratiker Xenophanes (gestorben nach 478 v. Chr.). Bereits dieser Autor formulierte den engen Zusammenhang von allmählichem

9 In der Edition von Wolgast S. 161 (dort als ein Zitat von Kritikern des Autors ohne Nachweis). - In dem unterhaltsamen Briefwechsel zwischen Du Bois-Reymond und Anton Dohrn spielt die Rede von 1880 keine Rolle, wohl aber die von 1872: Emil du Bois-Reymond (1818-1896) - Anton Dohrn (1840-1909), Briefwechsel, hg. v. Ch. Groeben in Zusammenarbeit mit K. Hierholzer, Berlin u.a. 1985, S. 30-42 (es geht vor allem um eine italienische Übersetzung und deren Publikation). 
Wissenszuwachs, geduldiger Forschung und der dafür notwendigen Zeitdauer in epischer Form, also nicht in einem stren-

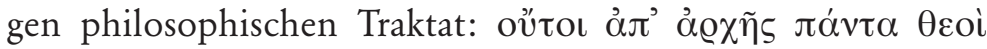

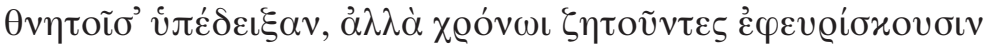

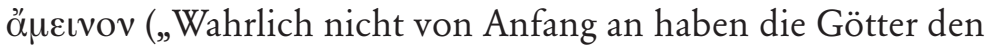
Sterblichen alles enthüllt, sondern allmählich finden sie suchend das Bessere " ${ }^{10}$ ). Entscheidend ist für unseren Zusammenhang hier einmal nicht die besondere und revolutionäre Form der Götterlehre, mit der Xenophanes vermutlich seine Umwelt ziemlich schockierte, sondern die Tatsache, daß hier der Fortschritt hin zu besserem Wissen (ö $\mu \varepsilon ı v o v)$ nicht an eine besondere göttliche Offenbarung gebunden wird, sondern an die Zeit

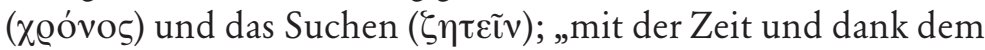
Suchen und Finden der Menschen geht es weiter und wird besser" ${ }^{\prime 11}$. Der spätantike Autor, der uns das Zitat über achthundert Jahre nach seiner Entstehung überliefert hat, ergänzt den frühen Text durch eine ganze Reihe von gleichlautenden offenbarungskritischen Aussagen späterer Epochen - offenkundig war die betreffende Position weit verbreitet: Es gibt kein menschliches Wissen, was nicht mit der Zeit und durch beharrliches Suchen erst erworben wird ${ }^{12}$.

Im platonischen Timaeus ist dagegen der Zusammenhang von Zeit und Erkenntnis viel grundsätzlicher gefaßt worden: $\mathrm{Zu}$ nächst erklärt Platon, daß erst die Wahrnehmung des Kreislaufs „von Tag und Nacht, Monaten und Umläufen von Jahren, Tag-

10 Xenophanes, frgm. 18 (DK 21 B 18, bei Stobaeus, eccl. I 8,2); vgl. dazu E.R. Dodds, The Ancient Concept of Progress, in: ders., The Ancient Concept of Progress and other Essays on Greek Literature and Belief, Oxford 1974, (S. 1-25) hier S. 4 f.; L. Edelstein, The Idea of Progress in Classical Antiquity, Baltimore 1967, S. 3-19; E. Heitsch, Das Wissen des Xenophanes, RhM 109, 1966, S. 193-235 und W. Jaeger, Die Theologie der frühen griechischen Denker, Stuttgart 1953, S. 50-68, vgl. auch J. Mittelstraß, Neuzeit und Aufklärung. Studien zur Entstehung der neuzeitlichen Wissenschaft und Philosophie, Berlin/ New York 1970, S. 54-58.

11 Heitsch, Das Wissen des Xenophanes, S. 220.

12 Vgl. dazu Johannes Stobaeus, eccl. I 8,2 (I, 94, 1-21). 
und Nachtgleichen und Sonnenwenden" für menschliches Er-

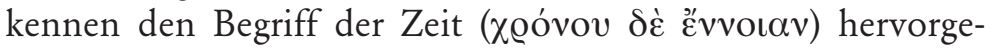
bracht hat. Erst dadurch ist aber „die Erforschung der Natur des

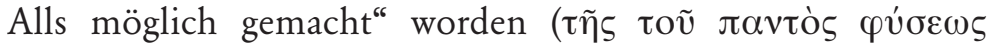
$\zeta \eta \dot{\tau \eta \sigma \iota v ~})^{13}$. Mit anderen Worten: Erst die Konzeptionalisierung der Zeit im Laufe von Zeit ermöglicht überhaupt grundlegenden Erkenntnisfortschritt, oder nochmals anders formuliert: Das magistrale Beispiel, an dem Platon seine These über die Bedeutung der Mathematik für den philosophischen Erkenntnisfortschritt demonstriert, ist die Zeit. Im Unterschied zu Xenophanes führt er freilich diese Ansicht nicht mit einer kritischen Note gegen die Götter ein, sondern nennt die Möglichkeit, durch den Gesichtssinn den erwähnten Kreislauf von Tag und Nacht wahrzunehmen, Zeit zu konzeptualisieren und so Wissen über die Natur des Alls zu erweben, das größte Geschenk aller Zeiten, das die Götter gegeben haben. Mit dieser Formulierung schließt übrigens auch Ciceros Übersetzung des platonischen Dialogs ins Lateinische ${ }^{14}$. Man könnte sich diese Bedeutung der Zeitlichkeit für das Erkennen übrigens ja auch noch an einem Detail platonischer Philosophie deutlich machen, an das philosophisch anzuknüpfen heute vermutlich nicht gerade leicht fällt: Wenn Platon Lernprozesse der Erkenntnis im Blick auf die Seele als Erinnerung an die Ge-

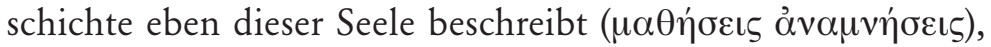
dann ist auch hier wieder die Zunahme von Wissen ganz eng mit der Erkenntnis von Zeitlichkeit verbunden ${ }^{15}$. Hier wäre

13 Platon, Tim. 47 A/B; hier zitiert nach H. Dörrie, Die geschichtlichen Wurzeln des Platonismus. Bausteine 1-35: Text, Übersetzung, Kommentar, aus dem Nachlaß hg. v. A. Dörrie (Der Platonismus in der Antike 1), Stuttgart-Bad Cannstatt 1987, S. 238.

14 Abgedruckt bei Dörrie, Platonismus in der Antike 1, S. 238.

$15 \mathrm{Vgl}$. das Zitat der griechischen Wortverbindung bei Tert., an. 23,6 (zitiert und kommentiert bei: H. Dörrie †/M. Baltes, Die philosophische Lehre des Platonismus. Von der „Seele“ als der Ursache aller sinnvollen Abläufe, Bausteine 169-181: Text, Übersetzung und Kommentar [Der Platonismus in der Antike 6.2], Stuttgart-Bad Cannstatt 2002, S. 12 154-163). 
eigentlich auch der gebotene Ort, über christliche Autoren und vor allem über Augustinus und das elfte Buch seiner Bekenntnisse - zu sprechen. Ich belasse es bei dem Hinweis, daß auch hier das „sinnliche Durcheilen früherer Zeiträume“ die Voraussetzung tieferer Selbst- und Gotteserkenntnis is ${ }^{16}$.

Natürlich stellt sich bei der Annahme immanenter Progressivität des Wissens im Horizont der Zeitlichkeit sofort die Frage nach dem Ziel bzw. dem Abschluß des Prozesses - also im Kern das bereits erwähnte Problem der Grenze des immerwährenden immanenten Fortschritts. Es wird in der Antike besonders deutlich an einer bekannten Aussage, die sich nach dem Bericht Ciceros im Protrepticus des Aristoteles befand, einer Einführungsschrift in die philosophische Lebenshaltung. Aristoteles klagte nach dem Referat Ciceros die veteres philosophos an, „die gemeint hätten, durch ihre Leistung sei die Philosophie vollendet worden, sie wären entweder sehr dumm oder sehr redselig gewesen; er (sc. Aristoteles) sähe indes, daß die Philosophie, weil in wenigen Jahren ein gewaltiger Zuwachs hinzugekommen sei, in wenigen Jahren völlig abgeschlossen sein werde“"17. Auch wenn inzwischen vermieden wird, den Protrepticus des Aristoteles als "Jugendschrift“ des Philosophen zu bezeichnen, bleibt selbst angesichts von fünfzehn Jahren Tätigkeit in der Akademie nur schwer verständlich, warum den alten Philosophen, die die Vollendung der Philosophie behaupteten, Dummheit oder Geschwätzigkeit vorgeworfen wird, aber Aristoteles von eben diesem Ziel nur wenig Jahre - brevi tempore - entfernt sein will. Es geht mir im Augenblick aber gar nicht um die historische Frage,

16 Aug., conf. XI 13,15; vgl. K. Flasch, Was ist Zeit? Augustinus von Hippo. Das XI. Buch der Confessiones. Historisch-philosophische Studie. Text - Übersetzung - Kommentar, Frankfurt/M. 1993, S. $248 \mathrm{f}$.

17 Cicero, Tusc. III 69: Itaque Aristoteles veteres philosophos accusans, qui existumavisset philosophiam suis ingeniis esse perfectam, ait eos aut stultissimos aut gloriosissimos fuisse; sed se videre, quod paucis annis magna accessio facta esset, brevi tempore philosophiam plane absolutam fore ( $=$ frgm. 53 Rose $^{3}=8$ Ross); vgl. auch O. Gigon, Cicero und Aristoteles, in: Hermes 87 (1959), S. 143-162, insbesondere S. $158 \mathrm{f}$. 
ob Cicero hier Aristoteles präzise verstanden hat ${ }^{18}$, sondern nur um den systematischen Hinweis, daß von der dem Wissen immanenten Progressivität - wenn man sie denn behaupten will tatsächlich, wie von Volker Gerhardt vorgeführt, „funktionale Unendlichkeit" 19 angenommen werden muß, um nicht in die Aporie des Aristoteles - oder sagen wir vorsichtiger: die von $\mathrm{Ci}_{-}$ cero dem Aristoteles zugeschriebene Aporie zu geraten. Solche Argumente haben freilich eine Reihe kluger neuzeitlicher Wissenschaftler auch nicht daran gehindert, das Erreichen der Vollständigkeit allen Wissens anzukündigen und das Ende der Wissenschaft für diesen Moment zu proklamieren ${ }^{20}$.

\section{Eine Erwägung zu den Pessimisten}

Wenn auf die eben beschriebene Weise aber der immanente oder gar der entscheidende Fortschritt, auf den Wissen hindrängt, eng mit der Zeit bzw. bei Platon mit der Konzeptualisierung der Zeit verbunden wird, dann drängt sich natürlich sofort die Frage auf, ob auch die kritische Stellungnahme gegen ein allzu optimistisches Fortschrittsmodell mit einer Reflexion über Zeit verbunden war. Der vorhin erwähnte Vorsokratiker Xenophanes hilft an dieser Stelle leider nicht weiter, weil sich seine kritische Wendung gegen sicheres Wissen ( in einem Fragment findet, indem gerade keine zeitliche Dynamik die pessimistische Pointe unterbricht, daß „scheinbares

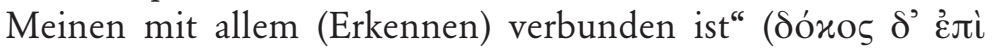

18 Daran könnte man zweifeln, vgl. ders., soph. ref. 34183 b. - Auf der anderen Seite begrenzt Aristoteles den Fortschritt der Theorie u.a. durch das Argument, daß die Zahl der Axiome begrenzt ist: met. II 1993 b 12-31; e.n. I 31094 b 12-14 sowie met. II 3995 a 15-20,

19 Gerhardt, Wissen in der Zeit, im vorliegenden Band, S. 53.

20 Hinweise bei J. Mittelstraß, Das Undenkbare denken. Über den Umgang mit dem Undenkbaren und Unvorstellbaren in der Wissenschaft (Preprint 105 des Max-Planck-Institutes für Wissenschaftsgeschichte), Berlin 1998, S. 3 Anm. 3-5. 


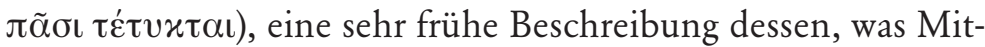
telstraß jüngst die dem wissenschaftlichen Verstand eigenen Unzulänglichkeiten nannte ${ }^{21}$. Man muß vielmehr auf Philosophen sehen, die den allgemeinen tiefsitzenden Pessimismus, der die griechische Kultur ungeachtet der großen zivilisatorischen Fortschritte prägte 22 , in der Erkenntnistheorie verankerten. Studiert man entsprechende Texte der verschiedenen skeptischen Schulen und schulfreien Philosophen der Antike, so wird deutlich, daß „Zeit“ zwar keine besondere systematische Rolle für eine solche skeptische Position spielt, aber doch gern von Skeptikern mit der „Zeit“ argumentiert wird: nibil posse percipi ( $\pi \dot{\alpha} v \tau \alpha \dot{\alpha} x \alpha \tau \alpha \dot{\lambda} \eta \pi \tau o v)$, nichts kann erfaßt und begriffen werden, nicht einmal dieser Satz selbst ${ }^{23}$ - eine solche radikal skeptische Position in der Tradition des Arkesilaos muß systematisch gesehen von jeder Thematisierung der Zeit absehen und allzumal von der Historisierung dieser Position. Für sie wird, soweit ich sehe, auch kaum bistorisch mit Rückschritten und Stagnationen argumentiert. Es heißt einfach: ,Seit Jahr-

21 Siehe oben.

22 K.D. Bracher, Verfall und Fortschritt im Denken der frühen römischen Kaiserzeit. Studien zum Zeitgefübl und Geschichtsbewußtsein des Jabrbunderts nach Augustus (Studien zu Politik und Verwaltung 21), Wien/Köln 1987 (= Diss. phil., Tübingen 1948/1949); E.R. Dodds, Der Fortschrittsgedanke in der Antike und andere Aufsätze zu Literatur und Glauben der Griechen, ins Deutsche übertragen von K. Morgenthaler (BAW. Forschung und Deutung A), Zürich u.a. 1977 (= ders., The Ancient Concept of Progress, Oxford 1972); Edelstein, The Idea of Progress in Classical Antiquity (wie Anm. 10), S. 17, 53 f.; C. Meier, Art. Fortschritt II. Fortschritt in der Antike, Geschichtliche Grundbegriffe II, Stuttgart 1975, S. 353-363; ders., Ein antikes Äquivalent des Fortschrittsgedankens, in: HZ 226 (1978), S. 265-316.

23 Vgl. die Debatte über diesen Satz bei Cicero, Luc. 28 und M. Frede, Stoics and Skeptics on Clear and Distinct Impressions, in: M. Burnyeat (Ed.), The Skeptical Tradition (Major Thinkers Series 3), Berkeley 1983, (S. 65-93) hier S. 86-92 bzw. ders., The Skeptic's Two Kinds of Assent and the Question of the Possibility of Knowledge, in: R. Rorty u.a. (Eds.), Philosophy in History. Essays in the Historiography of Philosophy, Cambridge 1984, S. 255-278. 
hunderten hat die Philosophie keine Fortschritte gemacht' (§ $15 \mathrm{f}$.); ,Es ist gar nichts geschehen, es wurde nichts Neues geforscht' (§ 16f.). Und doch spielt, wie Ciceros Dialog Lucullus zeigt, die „Zeit“ und ihre Konzeptionalisierung für Skeptiker eine erhebliche Rolle, weil Befürworter und Gegner der radikal skeptischen Position in der Neuen Akademie bei Cicero um die

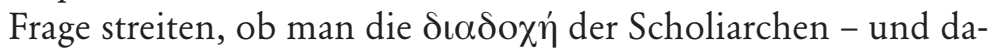
mit letztlich Platon - wirklich zur Autorisierung dieser Ansicht

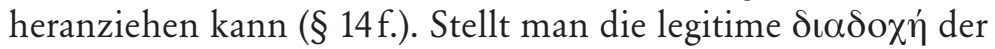
skeptischen Neuen Akademie in Frage, dann bleibt nur, ihr Zerstörung der eigentlich auf Fortschritt angelegten Philosophie vorzuwerfen, wie dies im Dialog auch geschieht (§ 15). Halten wir also fest: Auch wenn für die pessimistische radikale Bestreitung immanenter Fortschrittlichkeit des Wissens die „Zeit“ und ihre Konzeptualisierung keine zentrale systematische Rolle spielen, kommt die betreffende philosophische Richtung doch nicht darum herum, sich mit dem Thema auseinanderzusetzen und einen Autoritätsbeweis zu führen.

\section{Eine Erwägung zu denen, die sich nicht entscheiden können}

Wir haben also gesehen, daß sowohl die Optimisten, die mit dem Wissen unmittelbar einen beständigen, funktional infiniten Progreß eben des Wissens verbinden, als auch die Pessimisten, die mit ständigen Stagnationen und Rückschritten rechnen, bereits in der Antike „Zeit“ im doppelten Sinne ablaufender (Lebens-)Zeit und als Vorstellung (ह้vvoı $\alpha$ ) begriffener Zeit jeweils als zentrales Argument für ihre Sichtweise heranzogen, wenn auch an sehr verschiedenen systematischen Stellen. Ein neuzeitlicher Historiker hat Schwierigkeiten, sich einfach für eine der beiden Positionen zu entscheiden, weil er die Stagnationen und Rückschritte viel zu stark wahrnimmt, als daß er sie ignorieren könnte. Wie sagt Mittelstraß so schön: „Im übrigen erfindet sich das wissenschaftliche Denken gewissermaßen ständig neu, reali- 
siert sich in seinen Konstruktionen und vernichtet sich mit seinen Konstruktionen. Phönix ist das Symbol der Wissenschaft" ${ }^{\text {"24. }}$ Die Autorität Isaac Newtons (1643-1727) hat nun einmal die Weiterentwicklung der von Christian Huygens (1629-1695) entdeckten Theorie der Wellennatur des Lichts um etwa ein ganzes Jahrhundert verzögert, die durch Louis de Broglie (1897-1982) und David Bohm (1917-1992) aufgestellte „Theorie der verborgenen Parameter" als Interpretation der Quantenmechanik hat sich weitgehend als haltlos erwiesen ${ }^{25}$. Auf der anderen Seite machen

24 Mittelstraß, Das Undenkbare denken (wie Anm. 20), S. 19.

25 Den Darmstädter Physikern Freder Beck und Achim Richter danke ich für präzise Informationen zu diesen beiden Punkten und ergänze die Darstellung im Haupttext durch folgende, aus ihrer Feder stammende Erläuterungen: Newton propagierte die Korpuskulartheorie des Lichts: Licht besteht aus Teilchen, die eine bestimmte Farbe besitzen. Gegen die von Huygens vertretene Wellentheorie wandte er ein, daß damit die geradlinige Ausbreitung des Lichts (in der geometrischen Optik) nicht zu verstehen sei. Newtons Autorität bewirkte, daß die Ansichten von Huygens nicht mehr sehr beachtet wurden und sich damit die Weiterentwicklung der Wellentheorie um etwa ein ganzes Jahrhundert verzögerte. Es ist eine Ironie der Geschichte der Physik, aber auch ein Zeichen ihrer Kontinuität, daß die Quantentheorie dem Licht beide Eigenschaften, Welle und Teilchen (Lichtquanten), zuerkennt! Die Quantentheorie hat sich nur mühsam entwickelt. Das Bohrsche Atommodell basiert auf der klassischen Mechanik mit ad hoc Zusatzforderungen, die auf der Basis der klassischen Physik unverständlich bleiben. Bohr und Sommerfeld haben später Quantisierungsvorschriften angegeben, die die Bohrschen Zusatzforderungen in die Mechanik einbauen. Erst Werner Heisenberg zeigte durch seine ,Unschärferelation', daß den klassischen Observablen, wie z.B. Ort und Impuls eines Teilchens, keine objektive Bedeutung zugeordnet werden kann. Die Heisenbergsche und Schrödingersche Quantenmechanik beinhaltet den Indeterminismus der mikroskopischen Welt. Da unsere gesamten Erfahrungen und Denkgewohnheiten auf Beobachtungen in der makroskopischen Welt beruhen, ist eine aus der Gegenwart resultierende unbestimmte Zukunft dem menschlichen Geist nur schwer vermittelbar. „Gott würfelt nicht“, wie Einstein es formulierte. Von einem Pionier der Quantentheorie, Louis de Broglie, und später David Bohm stammt daher die ,Theorie der verborgenen Parameter', die besagt, daß zwar nach wie vor die klassischen Observablen objektive Werte besitzen, die allerdings der Messung nicht zugänglich sind, was zu der scheinbaren Unbestimmtheit der quantenmechanischen Größen, die Meßwerten entsprechen, führt. Die 
gerade diese Beispiele für Rückschritte und Stagnationen in der physikalischen Theoriebildung deutlich, daß Irrwege in den Naturwissenschaften in aller Regel nur eine begrenzte Zeit bestehen können, bis neue Messungen oder andere Prüfverfahren ihre Unhaltbarkeit aufzeigen. Wieder ist die Kategorie „Zeit“ für das Verständnis der dem Wissen immanenten Dynamik von elementarer Bedeutung: So, wie dem impliziten Fortschritt von Volker Gerhardt funktionale Ewigkeit zugewiesen wird, muß dem Rückschritt bzw. der Stagnation mindestens eine bestimmte Zeit zugewiesen werden (wenn nicht mehr bzw. anderes). Im wissenschaftlichen Idealfall gibt übrigens der theoretische Status, der von einer Theoriebildung behauptet wird, den eben beschriebenen Zwischenstatus allen Wissens zwischen Fortschritt, Stagnation und Rückschritt möglichst präzise wieder ${ }^{26}$.

Eine solche, möglichst präzise Selbstauskunft über den Status eigenen Wissens ist natürlich auch wieder kein Spezifikum derjenigen neuzeitlichen Wissenschaftler, die über Grundkenntnisse einer Wissenschaftstheorie und -methodik verfügen wie du Bois-Reymond schon 1880 zutreffend behauptet hatte, gibt es selbstverständlich auch antike Beispiele. Es ist vor einiger Zeit nachgewiesen worden, daß der kaiserzeitliche Mediziner und Philosoph Galen aus Pergamon eine solche präzise Selbstdiagnose der unterschiedlichen Status seiner Wissensbereiche versucht hat ${ }^{27}$. Medizinische Analyse und philosophi-

Theorie der verborgenen Parameter hat sich durch Experimente, die erst in jüngster Zeit mit Hilfe der Laseroptik möglich wurden, als weitgehend haltlos erwiesen. Sie war insofern ein gravierender Irrweg, als sie die Akzeptanz einer prinzipiell unbestimmbaren Zukunft im Mikroskopischen verzögerte.

26 So Beck/Richter: Theorien, wie derzeit das, Standardmodell ' der Elementarteilchenphysik, die bisher noch nicht durch Meßergebnisse widerlegt sind, sind daher als mathematisches Bild ,wahr', aber nicht als unumstößliche Wahrheit im objektiven Sinn, sondern als bisher widerspruchsfreies Abbild des, wahren' Naturgeschehens.

27 R.J. Hankinson, Galen's Concept of Scientific Progress, ANRW II.37.2., Berlin/ New York 1994, S. 1775-1789; allgemein zu Galen auch Edelstein, The Idea of Progress in Classical Antiquity (wie Anm. 10), S. 145-159. 
sche Methodenlehre greifen hierbei eng ineinander ${ }^{28}$ : Galen nimmt zwar für sich in Anspruch, durch eigene Beobachtungen und Diagnosen das medizinische Wissen erweitert zu haben ${ }^{29}$, auch denkt er, daß die peripatetische und stoische Logik unvollständig sind und vervollständigt werden könnten (und hat entsprechend Beiträge $\mathrm{zu}$ den logischen Schlußfiguren vorgelegt $)^{30}$. Aber gleichzeitig markiert er präzise den lediglich approximativen Charakter seiner medizinischen Wissenschaft, der quasi automatisch auch Stagnation und Rückschritte impliziert. Außerdem beobachtet er zusätzlich Rückschritt einzelner Disziplinen, beispielsweise einen starken Verfall der anatomischen Wissenschaft ${ }^{31}$. Daher verwendet Galen auch kein radikales Fortschrittsmodell, um die Arbeit am medizinischen Wissen zu beschreiben, vielmehr besteht für ihn Fortschritt in einer bestimmten Form des Rückschritts oder Rückgriffs - er orientiert

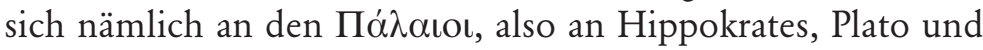
Aristoteles, dies freilich nicht sklavisch ${ }^{32}$. Dabei kommt ihm

28 Der Mediziner soll am besten auch Philosoph sein (Quod optimus medicus sit quoque philosophus I 53-63).

29 Galen sieht sich als „innovator in medical science“ (Hankinson, Galen's Concept, S. 1779); cf. De causis Procatarticis I 1 (CMG Suppl II, Bardong 1,3-11: et augeri quidem in eorum adinventionibus secundum illa tempora ab binc maxime proveniebat. Die Medizin schreitet durch sorgfältige Entdeckungen erkennbar voran (vet. med. 2, was Galen aber nicht bekannt war), vgl. auch Galen, In Hippocratis de auctorum morborum victu XV 433-438: Die Verschiedenheit verschiedener Therapien, angeboten durch verschiedene Ärzte, zeigt, daß Medizin doch nicht besser als Divination ist.

30 De Libris propriis XIX 46/47.

31 De Plenitudine VII 590/591; De Methodo Melendi (X 206; 806/807); De Crisibus (IX 657); De Praesagitatione ex Pulsibus (IX 216); In Hippocratis Prognosticum XVIII B 256. - Zum Verfall der Anatomie vgl. AA II 280-282, zu eigenen Entdeckungen AA II 659.

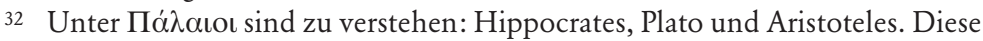
„Alten“ haben genuine Fortschritte gemacht, ihre Nachfolger versuchen es lediglich, weil sie Ehre bekommen wollen. Es gibt daher ein „goldenes Zeitalter" griechischer Wissenschaft (1780). De facultatibus naturalibus II 5 8,27,38,52,116,130-132,150; De usu partium I 3. Galen folgt der „Methode 
eine besondere pädagogische Verantwortung zu, weil die meisten Leute zu dumm sind, um zu verstehen, was die Alten ge-

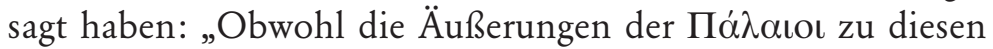
Zusammenhängen zutreffend waren, haben sie ihre Fälle nicht mit einem argumentativen Nachweis verbunden; sie haben nämlich nicht vermutet, daß es Sophisten geben könnte, die offenkundigen Fakten widersprechen würden. Deswegen habe ich selbst versucht, meine Argumente in einer Richtung zu wählen,

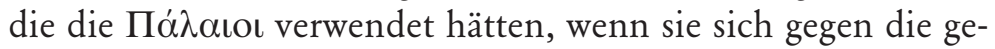
wandt hätten, die die besten Leistungen der Wissenschaft zunichte machen wollen“33.

An dieser Stelle können wir unseren fragmentarischen Durchgang durch antike Stimmen zum Problem einer immanenten Progressivität des Wissens im Horizont der Zeitlichkeit einstweilen abbrechen. Wir hatten ja eingangs gefragt, ob ein solcher archäologischer Blick helfen könnte, gegenwärtige Diskussionen über implizite Progressivität, über Fortschritt, Stagnation und Rückschritt in der Wissenschaft präzisier zu führen. Es ist vermutlich deutlich geworden, daß vor allem die in den letzten Jahrzehnten nur sehr partiell - beispielsweise hier im Hause studierte antike Fachliteratur dabei helfen könnte, differenzierte Konzeptionen über das Verhältnis von Fortschritt, Stagnation und Rückschritt auszuarbeiten, die die drei praktischen Grenzen bei Mittelstraß (Irrtumsgrenzen, ökonomische und ethische Grenzen) zugleich präzisiert und vielleicht ergänzt. Schließlich liegen diese Grenzen auch auf sehr verschiedenen Ebenen: Ökonomische Grenzen werden dem Wissenschaftler gesetzt und mit viel Geschick bei der Einwerbung von Drittmitteln vermag er sie zu verschieben; ethische Grenzen sind ihm

des Hippokrates“ (Meth. Med. X 309, 346). Vgl. auch G. Patzig, Veritas filia temporis?. Ein Vorschlag zur Differenzierung, in: Zukunft des Wissens, (S. 60-73) hier S. 64.

33 Übersetzung nach Hankinson, Galen's Concept, 1783 = fac. nat. II 178-180. 
wohl auch gesetzt, aber er sollte sie sich vor allem selbst setzen, denn was nützt die strengste gesetzliche Normierung, wenn die Grenze nicht akzeptiert, sondern verschoben wird; ob er die ihm gesetzten Irrtumsgrenzen überwinden kann, kann man mit Fug und Recht bezweifeln.

Für den Laienblick eines Historikers wirken die meisten gegenwärtig vertretenen Modelle über die immanente Entwicklungsdynamik des Wissens noch sehr grobkörnig, wenn sie beispielsweise den Fortschritt an eine einzige Form der Entwicklung - nämlich die der Revolution - binden oder sehr schlichte Teleologien des Fortschritts (beziehungsweise des Rückschritts) verwenden. Mir scheint, daß wir eine engere Verbindung von Wissenschaftstheorie und Wissenschaftsgeschichte brauchen, um die verschiedenen Konstellationen des Verhältnisses zwischen Wissen und Zeit in Zukunft etwas feinkörniger beschreiben oder gar modellieren zu können. Ich werde das heute nachmittag selbstverständlich nicht mehr versuchen, denn dies näher auszuführen, würde bedeuten, einen neuen Vortrag zu halten. Aber ich erlaube mir als evangelischer Theologe am Schluß den Hinweis, daß eine, wenn nicht die zentrale Voraussetzung für ein solches ausgewogenes Verhältnis von Fortschritt, Stagnation und Rückschritt in der Wissensentwicklung darin liegt, daß Wissens- und Glaubenswelten nicht unter der Hand verwechselt werden beziehungsweise - um ein wenig provokativ zu schließen - nicht im einundzwanzigsten Jahrhundert Wissenschaften oder Wissenschaftskulturen versuchen, die mit Absolutheitsanspruch bewehrten Positionen einzunehmen, die bis weit in die Neuzeit hinein Religion und Theologie besetzt haben ${ }^{34}$. Sonst würde beispielsweise die Ge-

34 Diese Entwicklung ist freilich schon längst im Gange: Vgl. neben dem oben zitierten Vortrag von D. Simon jetzt einen anderen Eröffnungsvortrag: J. Renn, Wissenschaft als Lebensorientierung. Eine Erfolgsgeschichte?, in: Leben. Verständnis - Wissenschaft - Technik. XII. Europäischer Kongreß für Theologie, 2002 Zürich (im Druck). 
fahr drohen, daß der immense Fortschritt des Wissens in den sogenannten „Lebenswissenschaften“ mit einem bestürzenden wissenschaftstheoretischen Rückschritt einhergeht. Und das kann eigentlich niemand wollen, am wenigsten die Lebenswissenschaftler selbst. 